\title{
Uso de técnicas de procesamiento de lenguaje natural para la detección de requerimientos de software
}

\section{Use of natural language processing techniques for software requirements detection}

DOI: $10.46932 /$ sfjdv2n5-072

Received in: Oct 1st, 2021

Accepted in: Dec 30th, 2021

\author{
Oscar Giovanni Esparza Martínez \\ Universidad Autónoma de Zacatecas \\ Campus Siglo XXI, Edificio de Ingeniera de Software \\ Carr. Zacatecas-Guadalajara Km 6, C.P. 98160, Zacatecas, Zac., México. \\ E-mail: netgio2064@hotmail.com \\ Sodel Vázquez Reyes \\ Universidad Autónoma de Zacatecas \\ Campus Siglo XXI, Edificio de Ingeniera de Software \\ Carr. Zacatecas-Guadalajara Km 6, C.P. 98160, Zacatecas, Zac., México. \\ E-mail:vazquezs@uaz.edu.mx \\ Alejandro Mauricio González \\ Universidad Autónoma de Zacatecas \\ Campus Siglo XXI, Edificio de Ingeniera de Software \\ Carr. Zacatecas-Guadalajara Km 6, C.P. 98160, Zacatecas, Zac., México. \\ E-mail: amgdark@uaz.edu.mx
}

\section{RESUMEN}

En este trabajo se presenta la investigación sobre el uso de técnicas de Procesamiento de Lenguaje Natural (PLN) para la detección de requerimientos, según cifras del Project Management Institute (PMI) el 47\% del fracaso de los proyectos es causado por una deficiencia en el ejercicio de ingeniería de requerimientos (Siqueira Simões, 2017). Esto presenta un riesgo muy alto, por tal motivo la etapa de ingeniería de requerimientos puede llegar a poner en riesgo el desarrollo y entrega del producto de software.

Palabras clave: Ingeniería de Requerimientos, Procesamiento de Lenguaje Natural, Minería de Textos, Ingeniería de Software.

\begin{abstract}
In this work presents the research on the use of Natural Language Processing (PLN) techniques for the detection of requirements, according to figures from the Project Management Institute (PMI) 47\% of project failure is caused by a deficiency in the requirements engineering exercise (Siqueira Simões, 2017). This presents a very high risk, for this reason the requirements engineering stage can put the development and delivery of the software product at risk.
\end{abstract}

Keywords: Engineering Requirements, Natural Language Processing, Text Mining, Software Enginering. 


\section{INTRODUCCIÓN}

La ingeniería de requerimientos es una de las disciplinas dentro del desarrollo de software que a cobrado una gran importancia en los últimos años, debido a que puede llegar a reducir costos monetarios y de producción (Arias Chaves, 2007). Detectar errores en una etapa temprana durante la ingeniería de requerimientos, es mucho menos costoso que detectarlos en cualquier otra fase del desarrollo, incluso si el producto ya esta en producción. Si se lleva un buen proceso de análisis de requerimientos se puede lograr una buena representación de las necesidades del cliente, al igual que, resolver la problemática que aqueja al usuario, entregándole al final un producto de software que le de valor. Y como lo menciona Samame "Los proyectos no fallan al final, sino al principio".

El fracaso de los proyectos han aumentado a cifras alarmantes ocasionado por una deficiencia en el ejercicio de la ingeniería de requerimientos (Siqueira Simões, 2017). Algunos casos comunes son:

\footnotetext{
- $\quad$ El producto se entrega sin cumplir con los requerimientos especificados.

- $\quad$ La entrega final es un producto que no satisface al cliente, aunque este en tiempo y dentro del presupuesto.

- $\quad$ El proyecto incorpora requerimientos que no son manifestados por el cliente.

- $\quad$ La estimación de costo/esfuerzo se hace en base a un alcance equivocado ya que no considera algunas áreas funcionales y procesos de negocio.

- $\quad$ Fallas de comunicación sobre requerimientos, lo que resulta en la entrega de un producto defectuoso.

- Cambios innecesarios debido a la falta de atención por comprender correctamente las necesidades del cliente al principio.
}

El éxito o el fallo en un producto de software depende enteramente de la fase de requerimientos y al ser escritos en lenguaje natural pueden presentar defectos como ambigüedad, inconsistencia o legibilidad, y si no se atienden de manera correcta y a tiempo pueden llagar a que el producto presente desfases en tiempo como en dinero (Gacitúa Bustos, 2015).

Los defectos pueden aparecer en cualquier etapa del ciclo de vida de un producto de software, presentándose más comúnmente durante la construcción. Así mismo, un número significativo de defectos se originan desde los requerimientos, ya que las especificaciones no representan de manera acertada las necesidades del cliente (Siqueira Simões, 2017).

Tabla 1. Defectos potenciales por tamaño de sistema y origen

\begin{tabular}{|l|l|l|l|}
\hline \multicolumn{4}{|l|}{ Tamaño del sistema (en puntos de función) } \\
\hline & 100 & 1000 & 10000 \\
\hline Origen del defecto & Defectos potenciales (bugs/PF) & \\
\hline Requerimientos & 0.75 & 1.00 & 1.25 \\
\hline Arquitectura & $\mathbf{0 . 1 0}$ & $\mathbf{0 . 2 5}$ & $\mathbf{0 . 5 0}$ \\
\hline Diseño & 1.00 & 1.25 & 1.50 \\
\hline Código fuente & 1.70 & 1.75 & 2.0 \\
\hline Material de pruebas & 1.50 & 1.85 & 2.00 \\
\hline Documentación & $\mathbf{0 . 6 5}$ & $\mathbf{0 . 7 0}$ & $\mathbf{0 . 7 5}$ \\
\hline Base de datos & 2.00 & 2.75 & 3.00 \\
\hline Website & 1.50 & 1.75 & 2.00 \\
\hline Total & 9.20 & 11.30 & 13.00 \\
\hline
\end{tabular}


La Tabla 1 describe la cantidad de defectos potenciales que pueden generarse en diferentes etapas del desarrollo de software para diferentes tamaños del sistema.

A partir de la información de la tabla anterior, los defectos originados en la fase de requerimientos se encuentra en la posición 6, puesto que hay otras fases donde pueden encontrarse una mayor densidad de defectos, como por ejemplo, base de datos, código fuente y material de pruebas. A pesar de esto, los errores presentados durante la fase de ingeniería de requerimientos son los que muestran un mayor índice de complicaciones a la hora de corregirse por métodos tradicionales: pruebas y análisis estático, además de que pueden ser arrastrados a otras fases del ciclo de desarrollo.

Para ello nos es importante enfocarnos en una herramienta que involucre la minería de texto con técnicas de procesamiento de lenguaje natural para detectar los requerimientos de software sin ambigüedad.

\section{METODOLOGÍA}

El sistema será capaz de detectar los requerimientos dentro de un documento escrito en lenguaje natural, para reducir inconsistencias y ambigüedades al redactar un documento SRS, de esta manera lograr disminuir el índice de fallo en productos de software durante la fase de ingeniería de requerimientos.

a) En la primera etapa se realiza el proceso de investigar factores que provocan que un producto de software falle en la fase de requerimientos dentro de la industria.

b) Buscar información con temas relacionados con técnicas de procesamiento de lenguaje natural para la extracción de requerimientos y ver los trabajos que se han realizado con anterioridad.

c) Analizar datos relevantes de las investigaciones para tomar en cuenta que algoritmo se usan para el PLN

d) Investigar más a fondo los tipos de algoritmos de minería de texto que existen para seleccionar con los que trabajaremos.

e) Con base a lo investigado, definir que algoritmo de minería de texto y que técnica de PLN favorece mejor a nuestras necesidades y ayude a una mejor detección de los requerimientos.

f) Construir la herramienta de procesamiento de lenguaje natural y probar la extracción de los requerimientos.

\section{ANTECEDENTES}

En Buenos Aires, dentro de la Facultad de Ingeniería y Tecnología Informática, se realizo un investigación de extracción de requerimientos a través de técnicas de procesamiento de lenguaje natural y posteriormente se construirán los requerimientos dentro de un modelo Léxico Extendido del Lenguaje (LEL) (Litvak, Hadad, \& Doorn, 2016). Dentro de esta investigación, a parte de generar un documento de especificaciones de requerimientos a través de procesamiento de lenguaje natural, los autores buscaban eliminar las omisiones que el humano podría dejar pasar cuando se genera un documento SRS; otro punto importante que los autores detallan en su investigación es que su herramienta podría reconocer escritos de varios idiomas, esta característica genera un punto negativo como positivo, ya que detectaría los 
requerimientos de manera semiautomática, puesto que algunas oraciones no podrían ser leídas de forma automática y por ende ser validadas manualmente.

En Chile, dentro del Centro de Estudios de Ingeriría de Software (CEIS), se realizó una investigación de los desafíos que enfrenta el procesamiento de lenguaje natural para la detección de requerimientos, pues la utilización de estas técnicas pueden presentar problemas complejos al detectar dichos requerimientos en un sistema inmenso como lo es el lenguaje (Gacitúa Bustos, 2015). En esta investigación los autores rompen el paradigma del ser humano "buscar la optimización de todas nuestras tareas diarias" ya que mencionan que la comunicación e interacción que tiene el ingeniero de requerimientos con el cliente no lo puede remplazar un producto de software, en vista de que en una entrevista o cualquier técnica de levantamiento de requerimientos, el lenguaje coloquial o informal no es capaz de detectar estas herramientas.

En Irlanda, dentro del departamento de Informática y Sistemas de la Información, se realizo una investigación del rol que presenta hoy en día la práctica de procesamiento de lenguaje natural en la ingeniería de requerimientos, ya que al final de cuentas es una practica que favorece tanto al cliente como al ingeniero de requerimientos, pero esto no es así, ya que se busca que la interacción del cliente y el ingeniero favorezca a la mejor detección de las necesidades del cliente (Ryan, 1993). En esta investigación se plantea la misma problemática que la investigación pasada, no confiar en una herramienta de software que sustituya la interacción que tiene el ser humano, en este artículo lo plantea en otro ámbito, en este caso no depender abiertamente en un documento de texto como fuente de conocimiento, puesto que no se espera que el cliente redacte todas sus demandas o necesidades en una forma textual.

En Pakistán, dentro de varias asociaciones con varias universidades, se realizo una investigación donde apelaban la gran importancia de la extracción de los atributos de calidad a través de técnicas de procesamiento de lenguaje natural, ya que estos son importantes para un desarrollo exitoso, debido a que los atributos de calidad describen características del sistema y restricciones de diseño (considerados como Disponibilidad, Confidencialidad, Integridad, Usabilidad, etc.) (Asif et al., 2019). Dentro de este documento lo que se busca atacar primordialmente son los atributos de calidad ya que los encuentran como unos de los más importantes para el éxito de un producto de software, estos atributos de calidad en varias ocasiones llegan a ser omitidos porque no están definidos explícitamente y pueden estar ocultos dentro de un texto plano. En esta investigación se basan en el formato PDF para la extracción de requerimientos y atributos, lo interesante es que plantean incorporar las funcionalidades del sistema a través de una descripción completa de su funcionamiento del producto de software, incluyendo el diseño en texto, con fuentes e imágenes en formato de bits. Para esto usan un algoritmo de tamiz de paso múltiple y fragmentación de texto clasificado. 
En Reino Unido, Italia e Irlanda, con una asociación entre varias universidades realizaron una investigación sobre la detección de requerimientos especulativos a través de algoritmos de minería de texto, ya que la frecuencia con que se utiliza el lenguaje especulativo, puede llegar a presentar un alto riesgo de que los requerimientos puedan ser mal entendidos (Yang, De Roeck, Gervasi, Willis, \& Nuseibeh, 2012). Lo que se busca dentro de esta investigación es atacar los requerimientos tentativos o especulativos a través de técnicas de procesamiento de lenguaje natural, estos requerimientos pueden llegar a presentar comportamientos no deseados que no van acorde con las expectativas que el cliente desea, esto pasa en la mayoría de las ocasiones al transcribir de manera errónea las necesidades que requiere el cliente, esto al utilizar varios métodos de levantamiento de requerimientos, como puede ser la entrevista, lo cual puede generar dificultades posteriores en el desarrollo del producto de software. Otro punto que se aborda en la investigación pero de lado positivo de detectar los requerimientos especulativos, es que simplemente pueden dejar un espacio mucho más amplio en la interpretación del diseño, para que los desarrolladores puedan explorar más de una solución de los requerimientos especulativos.

\section{HIPOTESIS}

Al construir una herramienta de software donde se utilicen técnicas de minería de texto y procesamiento de lenguaje natural para el procesamiento de documentos de texto, dicha herramienta será capaz de detectar de manera semi-automatica la estructura morfológica, sintáctica y semántica que conforman los requerimientos, para que de esta manera el usuario tenga que validar que la salida los requerimientos sean correctos y de esta forma evitar las omisiones o las ambigüedades que se tienen al usar alguna técnica de levantamiento de requerimientos.

\section{ESTADO DEL ARTE}

1. Dentro del departamento de ciencias computacionales del norte de California (USA) se desarrolló una herramienta que ayudaría a extraer de manera efectiva los atributos de calidad dentro de un documentos de texto (uso de datos, manuales de instalación, reglamentos, solicitudes, especificación de requerimientos y de usuario), utilizando técnicas de procesamiento de lenguaje natural (Slankas \& Williams, 2013). El objetivo primordial al analizar los documentos, es que se pudiera extraer los atributos de calidad y de esta manera clasificarlos (p. ej. Seguridad, usabilidad, disponibilidad y confiabilidad).

Slankas y Williams desarrollaron un herramienta a la que le llamaron NFR Locator el cual les ayuda a clasificar y extraer parecidos existentes en las categorías de NFR. Se crearon 14 categorías en la cuales podrían caer los requerimientos no funcionales o atributos de calidad. 
1. Control de acceso

2. Auditoría

3. Disponibilidad

4. Capacidad y rendimiento

5. Legal

6. Apariencia

7. Mantenibilidad

8. Operacional

9. Privacidad

10. Recuperación de capacidad

11. Confiabilidad

12. Seguridad

13. Facilidad de uso

14. Otros.

La herramienta NFR Locator funciona a través de 2 pasos primordiales, extrae los requerimientos no funcionales en fases, se puede tomar cualquier documento de texto de cualquier proyecto, el procesamiento de lenguaje natural analiza el contenido y clasifica las oraciones en categorías, si el texto no concuerda con alguna categoría devuelve "no aplicable". Al final cada oración genera un gráfico Stanford Type Dependency Representation (STDR).

La Figura 1 representa un gráfico en forma de árbol que la herramienta NFR Location retorna, en este caso es el atributo de calidad para la oración "El sistema debe terminar sesión después de 30 minutos de inactividad".

Figura 1. Clasificador de sentencias

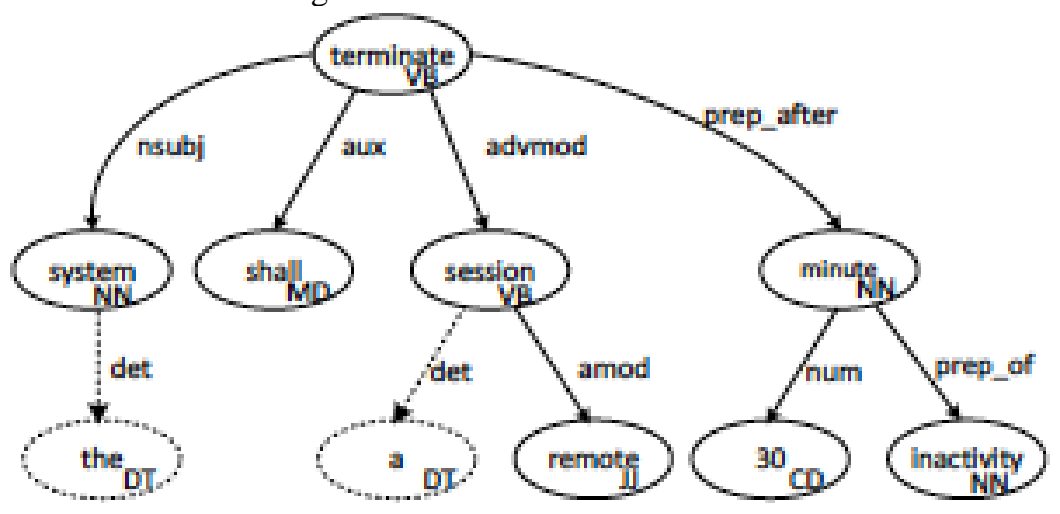

2. En la facultad de ciencias de la computación en Alemania se desarrollo un sistema el cual se extraen requerimientos de un documentó de texto simple y validaba la relación de estos entre los términos, puesto que los documentos de requerimientos a menudo son mal entendidos, por inconsistencia y por información incompleta (Kof, 2010). Lo que Leonid buscaba era una manera de poder detectar los requerimientos al modificar los conceptos, definirlos con precisión y con un simple glosario (lista de términos), a esto le llamo ontología, se define como una taxonomía (termino jerarquía). 
En la Figura 2 muestra los pasos para la extracción ontológica, el autor construyo un algoritmo en el cual se extraen los terminos, los agrupaba y hacia una construcción de taxonomía (como una jerarquía de agrupaciones) y minería de asociaciones, este procedimiento tenia que ser validado, mencionaba que el procedimiento de extracción automático no podría detectar en su totalidad los requerimientos sin inconsistencias.

Figura 2. Procedimiento de construcción de ontología

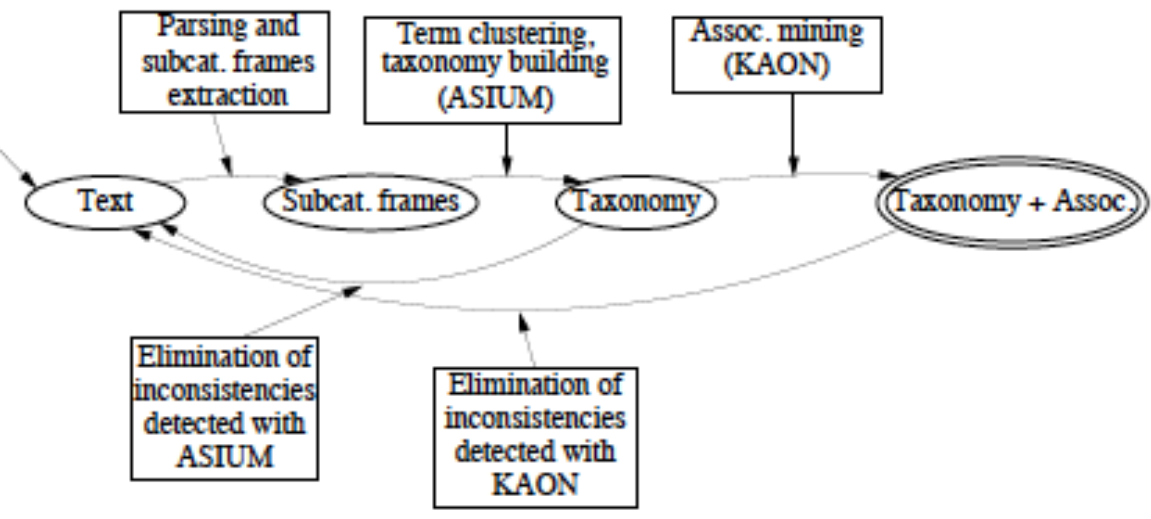

Lo que primero se hace es extraer los términos del texto de requerimientos, se analiza la oración y se descompone para relacionar las frases con el verbo principal. Después se agrupan los términos relacionados de las oraciones, como los requerimientos contenidos con el mismo contexto gramatical. Los conceptos construidos en el paso anterior se utilizan para la creación de la taxonomía en grupos, Kof utiliza la herramienta ASIUM para agrupar los términos y construir la taxonomía y durante este paso la terminología se valida con respecto a los sinónimos y así el usuario pueda identificarlos. Al final solo se hace una asociación entre 2 conceptos y se vuelve a repetir la operación para eliminar posibles inconsistencias.

3. Miembros y seguidores del Instituto de Ingeniería Eléctrica y Electrónica (IEEE) desarrollaron un sistema automatizado de conformidad de requerimientos a través de técnicas de procesamiento de lenguaje natural, el enfoque es una herramienta eficaz que pueda reducir la ambigüedad que existe en los requerimientos escritos en texto plano dentro de un documento o plantilla (Arora, Sabetzadeh, \& Briand, 2015). El objetivo de la herramienta para la extracción de los requerimientos esta enfocado en 2 áreas primordiales, la fragmentación de texto y la coincidencia de patrones en lenguaje natural. 
Para la fragmentación de texto, consta de un proceso de descomposición de oraciones en segmentos, de los cuales son agrupadas en frases sustantivas (puede ser un sujeto u objeto de un verbo) y frases verbales (un verbo).

La Figura 3(a) muestra un enunciado en texto plano del requerimiento, que al ser procesado por el Text chunking o la fragmentación de texto del PNL que desarrollaron los autores, se descompone la frase en elementos sustantivos y elementos verbales como lo muestra la Figura 3(b).

Figura 3. (a) Ejemplo de sentencia de requerimientos, (b) Fragmentación de texto

\section{$K:$ The information technology tools used in the design of systems performing safety functions shall be assessed for safety implications on the end-product.}

(a)

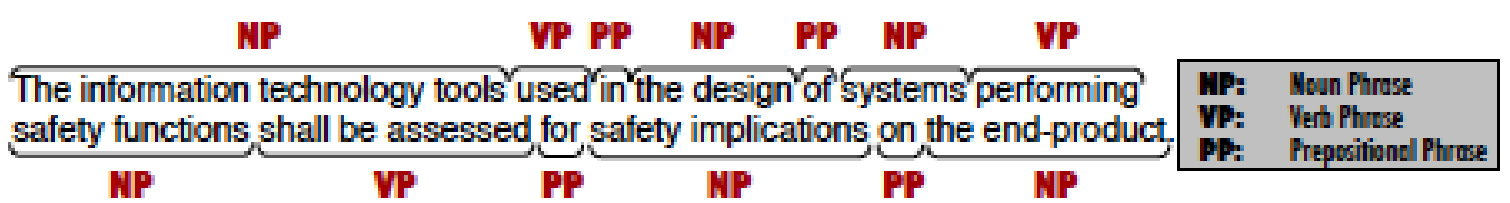

(b)

Cuando la fragmentación de texto es demasiado robusta (frases complejas o difíciles de identificar), se genera un árbol de análisis de segmentos, Arora, Sabetzadeh y Briand utilizaron Stanford Parser, un analizador de lenguaje natural de sentencias gramaticales para producir el árbol como el de la Figura 4.

Figura 4. Árbol de análisis de segmentos

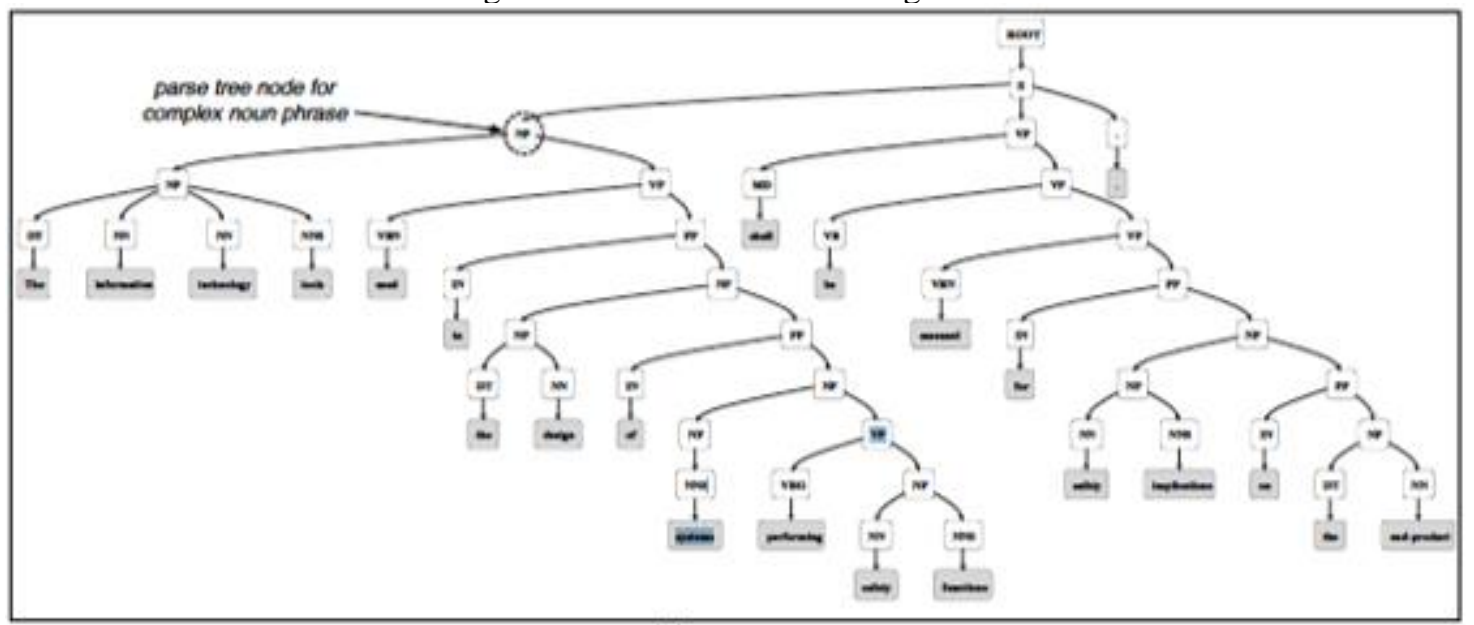

(c)

La Figura 5 representa el modulo de procesamiento de lenguaje natural, cuando se ingresa el documento donde se contienen los requerimientos en texto plano, el tokeneizer divide las entradas en tokens (este puede llegar a ser una palabra, un numero o un símbolo), el POS Tagger anota una etiqueta a 
cada token (la etiqueta puede ser un adjetivo, adverbio, sustantivo o verbo), el paso siguiente es identificar la etiqueta de acuerdo a la categoría, el paso final es agrupar el fragmento de la oración con respecto al módulo correspondiente a su etiqueta (frases sustantivas y verbales).

Figura 5. Patrón de coincidencia de lenguaje natural

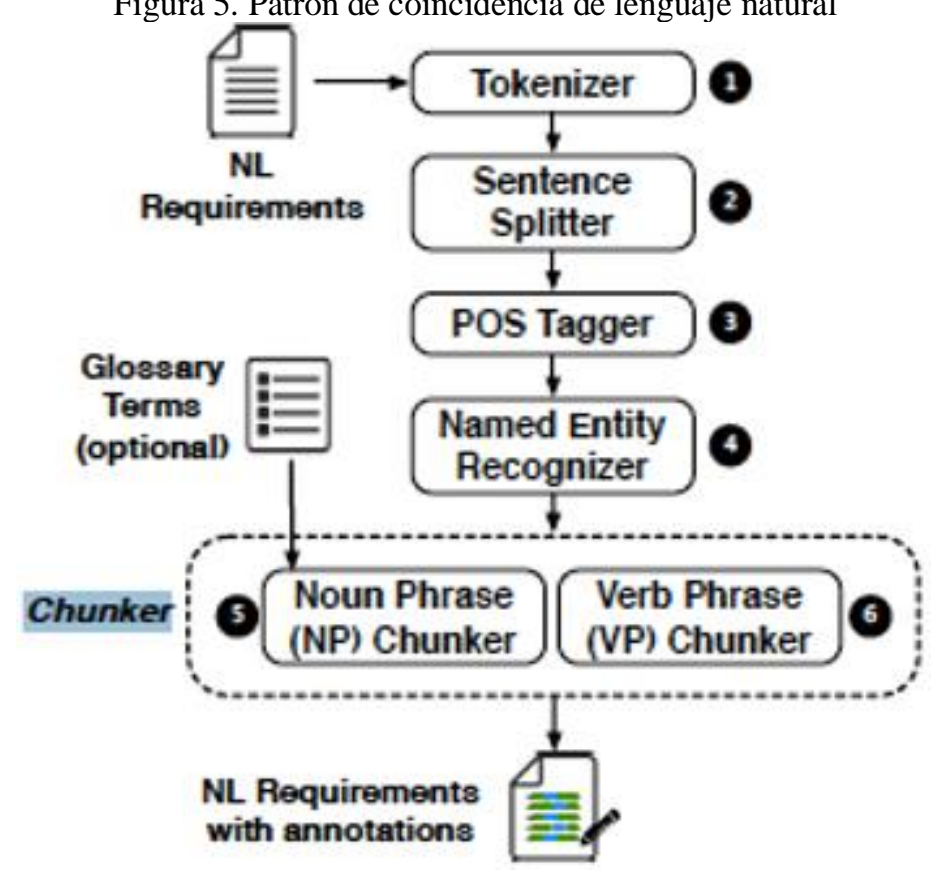

\section{RESULTADOS}

Al realizar esta investigación, nos dimos cuenta que hay un alto índice de defectos que llegan a presentarse en el área de requerimientos y al ser esta una área tan importante, pone en peligro el éxito de un producto de software, es por esto que nos vemos con la necesidad de generar un sistema que permita analizar textos en lenguaje natural para detectar de manera semiautomática especificaciones de requerimientos a través de minería de texto y técnicas de procesamiento de lenguaje natural, y de esta manera permita dar solución al problema del alto índice de defectos en el área de requerimientos, tales como ambigüedad, inconsistencia, carencia de legibilidad y omisiones de información, dando como resultado, presentar correctamente las necesidades de los clientes y usuarios sin llegar a generar gastos extras tanto de costos como de tiempo.

Al tener esto presente, fue necesario generar un investigación para detectar los mejores algoritmos y sistemas que implementan técnicas de procesamiento de lenguaje natural para la detección de requerimientos.

Dentro de la Tabla 2 observamos diferentes herramientas con distintos objetivos, desde mejorar la calidad de los requerimientos e historias de usuario, como también herramientas para la detección de 
ambigüedades e incertidumbre de los requerimientos. Unas de las herramientas mas sobresalientes son: QuARS, AQUSA y MaramaAI.

Quality Analyzer for Requirements Specifications (QuARS) es un producto de software desarrollado con la finalidad de facilitar la extracción de información estructurada y métricas para detectar inexactitudes y defectos lingüísticos que pueden causar problemas de ambigüedad usando el análisis de documentos de requerimientos a través de procesamiento de lenguaje natural (Lami, 2005). La herramienta QuARS trabaja con un PLN para mejorar la calidad de los requerimientos con análisis "no ambiguo" léxico y semántico, a partir de esto se determinaron 3 propiedades que la herramienta definen como mejora de los requerimientos (Expresividad, Consistencia y Completitud).

AQUSA es una mejora del framework QUS (Quality User Story) desarrollado por los mismos autores, este sistema mejora la calidad de las historias de usuario a través de una colección de 13 criterios en términos de sintaxis, pragmática y semántica, usando un analizador de procesamiento de lenguaje natural (Lucassen, Dalpiaz, \& Martijn, 2016). De los 13 criterios para la mejora de la calidad, algunos describen aspectos como, las historias deben de ser completas, incluyen un rol y un medio, las historias evitan términos que conduzcan a múltiples interpretaciones, cada historia es única y no hay duplicados o que detone una historia independiente, etc.

MaramaAI es una herramienta que tiene como objetivo la calidad de los requerimientos a través de verificar la coherencia, integridad y corrección de los requerimientos a través de un algoritmo heurístico y modelos formales que capturan los requerimientos en forma de interacciones abstractas y los transforma en una representación semiformal denominada casos de uso esenciales (Kamalrudin, Hosking, \& Grundy, 2010). Además de capturar los requerimientos, esta herramienta genera advertencias si se detecta una inconsistencia al capturar los requerimientos a través de una biblioteca de patrones de interacción esencial. 
Tabla 2. Comparación de herramientas

\begin{tabular}{|c|c|c|c|c|c|c|}
\hline $\begin{array}{l}\text { Nombre } \\
\text { Herramienta }\end{array}$ & Objetivo & Entrada & $\begin{array}{l}\text { Enfoque de } \\
\text { transformación }\end{array}$ & $\begin{array}{l}\text { Automatizació } \\
\text { n }\end{array}$ & Año & Exclusión \\
\hline Circe & $\begin{array}{l}\text { Calidad de } \\
\text { requerimient } \\
\text { os }\end{array}$ & $\begin{array}{l}\text { Documento de } \\
\text { requerimientos }\end{array}$ & $\begin{array}{l}\text { Regla } \quad \mathrm{de} \\
\text { Ontology based }\end{array}$ & $\begin{array}{l}\text { Semi- } \\
\text { automatico }\end{array}$ & 2006 & No \\
\hline NAI & $\begin{array}{l}\text { Ambigüeda } \\
\mathrm{d}\end{array}$ & $\begin{array}{l}\text { Documento de } \\
\text { requerimientos }\end{array}$ & $\begin{array}{l}\text { Regla de } \\
\text { Ontology based }\end{array}$ & Automático & 2010 & No \\
\hline QuARS & $\begin{array}{l}\text { Calidad de } \\
\text { requerimient } \\
\text { os }\end{array}$ & $\begin{array}{l}\text { Documento de } \\
\text { requerimientos }\end{array}$ & $\begin{array}{l}\text { Regla de } \\
\text { Ontology based }\end{array}$ & Automático & 20012004 & No \\
\hline CRF Tool & $\begin{array}{l}\text { Incertidumb } \\
\text { re }\end{array}$ & $\begin{array}{l}\text { Documento de } \\
\text { requerimientos }\end{array}$ & $\begin{array}{l}\text { Regla de } \\
\text { Ontology based }\end{array}$ & Automático & 2012 & No \\
\hline AQUSA & $\begin{array}{l}\text { Calidad de } \\
\text { las historias } \\
\text { de los } \\
\text { usuarios. } \\
\end{array}$ & $\begin{array}{l}\text { Historias } \\
\text { usuario }\end{array}$ & $\begin{array}{l}\text { Regla de } \\
\text { Ontology based }\end{array}$ & Automático & 2015 & $\begin{array}{l}\text { Tipo de } \\
\text { entrada }\end{array}$ \\
\hline T1' & $\begin{array}{l}\text { Ambigüeda } \\
\mathrm{d}\end{array}$ & $\begin{array}{l}\text { Documento de } \\
\text { requerimientos }\end{array}$ & $\begin{array}{ll}\begin{array}{l}\text { Basado } \\
\text { reglas }\end{array} & \text { en } \\
\end{array}$ & Automático & 2008 & No \\
\hline RAT & $\begin{array}{l}\text { Calidad de } \\
\text { requerimient } \\
\text { os }\end{array}$ & $\begin{array}{l}\text { Documento de } \\
\text { requerimientos }\end{array}$ & $\begin{array}{ll}\text { Basado } & \text { en } \\
\text { reglas } & \end{array}$ & Automático & 2009 & No \\
\hline Text2Test & $\begin{array}{l}\text { Calidad de } \\
\text { las historias } \\
\text { de } \quad \text { los } \\
\text { usuarios. } \\
\end{array}$ & Casos de Uso & Desconocido & Desconocido & - & No \\
\hline MaramaAI & $\begin{array}{l}\text { Calidad de } \\
\text { requerimient } \\
\text { os }\end{array}$ & $\begin{array}{l}\text { Documento de } \\
\text { requerimientos }\end{array}$ & $\begin{array}{l}\text { Regla de Pattern } \\
\text { based }\end{array}$ & $\begin{array}{l}\text { Semi- } \\
\text { automatico }\end{array}$ & 2011 & $\begin{array}{l}\text { No es } \\
\text { automático }\end{array}$ \\
\hline EuRailCheck & $\begin{array}{l}\text { Calidad de } \\
\text { requerimient } \\
\text { os }\end{array}$ & $\begin{array}{l}\text { Documento de } \\
\text { requerimientos }\end{array}$ & $\begin{array}{l}\text { Regla de } \\
\text { Ontology based }\end{array}$ & $\begin{array}{l}\text { Semi- } \\
\text { automatico }\end{array}$ & 2012 & $\begin{array}{l}\text { No es } \\
\text { automático }\end{array}$ \\
\hline UIMA & $\begin{array}{l}\text { Modelo de } \\
\text { caso de uso }\end{array}$ & $\begin{array}{l}\text { Descripción de } \\
\text { casos de uso }\end{array}$ & $\begin{array}{ll}\text { Basado en } \\
\text { reglas }\end{array}$ & Automático & 2009 & $\begin{array}{l}\text { Fuera de } \\
\text { ámbito }\end{array}$ \\
\hline DODT & $\begin{array}{l}\text { Calidad de } \\
\text { requerimient } \\
\text { os }\end{array}$ & $\begin{array}{l}\text { Documento de } \\
\text { requerimientos }\end{array}$ & $\begin{array}{l}\text { Regla de } \\
\text { Ontology based }\end{array}$ & $\begin{array}{l}\text { Semi- } \\
\text { automatico }\end{array}$ & 2011 & $\begin{array}{l}\text { No es } \\
\text { automático }\end{array}$ \\
\hline SREE & $\begin{array}{l}\text { Ambigüeda } \\
\text { d }\end{array}$ & $\begin{array}{l}\text { Documento de } \\
\text { requerimientos }\end{array}$ & $\begin{array}{l}\text { Regla de } \\
\text { Ontology based } \\
\end{array}$ & $\begin{array}{l}\text { Semi- } \\
\text { automatico }\end{array}$ & 2013 & No \\
\hline $\begin{array}{l}\text { Extraction of } \\
\text { OLAP req. }\end{array}$ & $\begin{array}{l}\text { Calidad de } \\
\text { requerimient } \\
\text { os }\end{array}$ & $\begin{array}{l}\text { Documento de } \\
\text { requerimientos }\end{array}$ & $\begin{array}{l}\text { Regla de Pattern } \\
\text { based }\end{array}$ & Automático & 2009 & $\begin{array}{ll}\text { Fuera de } \\
\text { ámbito }\end{array}$ \\
\hline HEJF & $\begin{array}{l}\text { Calidad de } \\
\text { requerimient } \\
\text { os }\end{array}$ & $\begin{array}{l}\text { Documento de } \\
\text { requerimientos }\end{array}$ & $\begin{array}{l}\text { Basado } \\
\text { reglas }\end{array}$ & Automático & 2014 & No \\
\hline
\end{tabular}

\section{CONCLUSIONES}

Para esta investigación se propuso construir una herramienta donde se utilicen técnicas de minería de texto y procesamiento de lenguaje natural a través de un producto de software para la lectura de documentos de texto, dicha herramienta detectará la estructura morfológica, sintáctico y semántico de los requerimientos, el usuario podrá validar si dichos requerimientos son correctos.

Se determinaron cuales serian las mejores herramientas para la detección de requerimientos a través de PLN y bajar a gran medida el índice tan alto que se tiene en fallos de sistemas por la fase de 
requerimientos. Como trabajo futuro se desarrollará la herramienta de procesamiento de lenguaje natural y probar la extracción de los requerimientos, teniendo como base las técnicas de PLN que ayuden a una mejor detección de los requerimientos.

\section{RECONOCIMIENTOS}

Expreso mi agradecimiento a la Universidad Autónoma de Zacatecas por el apoyo otorgado para la realización de esta investigación. 


\section{REFERENCIAS}

Siqueira Simões, G. (2017). La Importancia de la Ingeniería de Requerimientos. Retrieved June 9, 2019, from SG: Software Guru website: https://sg.com.mx/revista/54/la-importancia-la-ingenier-requerimientos.

Arias Chaves, M. (2007). La ingeniería de requerimientos y su importancia en el desarrollo de software. InterSedes: Revista de Las Sedes Regionales, p. 14. Retrieved from https://www.redalyc.org/articulo.oa?id=66612870011 [3] R. Zahmoul, R. Ejbali, M. Zaied, "Image encryption based on new Beta chaotic maps", Optics and Lasers in Engineering, Vol. 96, pp. 39-49. 2017.

Samame, R. (2015). Importancia de los Requerimientos del Proyecto. Retrieved June 9, 2019, from BSG Institute website: https://bsginstitute.com/bs-campus/blog/La-Importancia-de-los-Requerimientos-en-unProyecto-17.

Gacitúa Bustos, R. (2015). Procesamiento de Lenguaje Natural en Ingeniería de Requisitos: Contribuciones Potenciales y Desafíos de Investigación. 2-4.

Litvak, C. S., Hadad, G. D. S., \& Doorn, J. H. (2016). Procesamiento de Lenguaje Natural para Estudiar Completitud de Requisitos. WICC 2016 - XVIII Workshop de Investigadores En Ciencias de La Computación, Anales Electrónicos, 498-502.

Ryan, K. (1993). The role of natural language in requirements engineering. Proceedings of the IEEE International Conference on Requirements Engineering, 240-242. https://doi.org/10.1109/ISRE.1993.324852.

Asif, M., Ali, I., Malik, M. S. A., Chaudary, M. H., Tayyaba, S., \& Mahmood, M. T. (2019). Annotation of Software Requirements Specification (SRS), Extractions of Nonfunctional Requirements, and Measurement of Their Tradeoff. IEEE Access, 7, 36164-36176. https://doi.org/10.1109/ACCESS.2019.2903133.

Yang, H., De Roeck, A., Gervasi, V., Willis, A., \& Nuseibeh, B. (2012). Speculative requirements: Automatic detection of uncertainty in natural language requirements. 2012 20th IEEE International Requirements Engineering Conference, RE 2012 - Proceedings, 11-20. https://doi.org/10.1109/RE.2012.6345795.

Slankas, J., \& Williams, L. (2013). Automated extraction of non-functional requirements in available documentation. 2013 1st International Workshop on Natural Language Analysis in Software Engineering, NaturaLiSE 2013 - Proceedings, 9-16. https://doi.org/10.1109/NAturaLiSE.2013.6611715.

Kof, L. (2010). Natural Language Processing: Mature Enough for Requirements Documents Analysis? 91102. https://doi.org/10.1007/11428817_9.

Arora, C., Sabetzadeh, M., \& Briand, L. (2015). Automated Checking of Conformance to Requirements Templates using Natural Language Processing. (c), 1-25.

Arendse, B. (2016). A thorough comparison of NLP tools for requirements quality improvement. (2016) Faculty of Science Theses, (August). https://doi.org/M111.246231 [pii]10.1074/jbc.M111.246231 [doi]. Lami, G. (2005). QuARS: A Tool for Analyzing Requirements. CARNEGIE MELLON SOFTWARE ENGINEERING INSTITUTE. Retrieved from https://doi.org/10.1184/R1/6582770.v1

Lucassen, G., Dalpiaz, F., \& Martijn, J. (2016). Improving agile requirements : the Quality User Story framework and tool. Requirements Engineering, 21(3), 383-403. https://doi.org/10.1007/s00766-016-0250-X

Kamalrudin, M., Hosking, J., \& Grundy, J. (2010). MaramaAI: Automated and Visual Approach for Inconsistency Checking of Requirements. 393-394. https://doi.org/10.1109/RE.2010.54 